\title{
Electrical Response and Mechanical Behavior of Plasticized PVC Actuators
}

\author{
Hong Xia ${ }^{a}$, Takamitsu Uekiand ${ }^{\mathrm{b}}$ and Toshihiro Hirai ${ }^{\mathrm{c}}$ \\ Smart Materials Engineering, Faculty of Textile science and Technology, \\ Shinshu University, 3-15-1 Tokida, Ueda, Nagano 386-8567, Japan \\ as08t107@shinshu-u.ac.jp, bs05t104@shinshu-u.ac.jp, 'tohirai@shinshu-u.ac.jp
}

\begin{abstract}
Keywords: PVC gels, creep deformation, bending actuator, Young's modulus, compression, tensile,
\end{abstract} Maxwell stress.

\begin{abstract}
[Abstract] By changing gel and electrode shape, many different actuators could be invented. In the previous papers, we have reported that Maxwell stress generated in the PVC gels was expected as the results of polarization response of charges, and it mainly took place between the gel and the anode due to the accumulated negative charges on surface of the PVC gel near anode. Due to the existence of the Maxwell force, the PVC gel will be deformed asymmetrically and resulted in creep deformation along the anode. In this paper, we conducted some mechanical experiments and investigated the modulus of PVC gels with varied contents of plasticizer DBA. It was found that PVC gels were separated to two layers obviously after applied an electrical field. Near the anode, the layer (L1) was thinner and softer than other layer (L2) due to the transfer of plasticizer to the anode. This phenomenon was also confirmed by a fluorescence microscope. The displacement of gel in both sides near electrodes was different, and then a bending force was generated due to the force of electrical field. On the other hand, the recovery time of the deformed gel depended on the contents of plasticizer. The longer recovery time was observed for the lower content of plasticizer. The Young's modulus of PVC gels in tensile becomes small after applied an electrical field and the gels became to be deformed easily.
\end{abstract}

\section{Introduction}

In recent years, the smart materials have been interested and applied to various fields. As external stimuli, $\mathrm{pH}$, temperature, light, magnetic and electrical fields have been increased due to the untapped potential of such functional materials in a diverse range of nano and bio-related technologies [1]. Polyvinyl chloride (PVC) is one of the most versatile of commercial plastics that has low cost, light weight and is easy to product. Especially, PVC gels can convert electrical energy to dynamic mechanical work. Their unique deformation is significantly suitable for actuators development. As a material of actuator, the plasticized polyvinyl chloride (PVC gel) can exhibit fast response, low hysteresis and large deformation under an electrical field. For example, the bending actuator can be bent above $80^{\circ}$ of angle in several seconds [2]. The faucal variable lens can change $1 \mathrm{~mm}$ of faucal within 1 second [3]. In the past work, we have investigated and discussed the electrical response of PVC gels by some experiments, such as the reagents electrical response test, space charge measurement, force measurement and so on. The creep deformation of PVC gels was found to be the result of the Maxwell stress effect between electrodes and gel, due to the polarization of polymer and plasticizer and the charge/ions migrant to anode. But the relation of creep deformation and bending actuation was not very clearly.

In the present work, we prepare 4 kinds of PVC gels with difference plasticizer contents, and use thermal mechanical analyzer and fluorescence microscope to measure, observe and compare their mechanical properties under an electrical field.

\section{Experimental}

\section{Materials and preparation of specimens}

Plasticized PVCs were made from commercial PVC powder $(n=3700)$, solvent tetrahydrofuran (THF) and plasticizer DBA with 10:30, 10:50, 10:70 and 10:90 (wt \%). All of the materials were prepared by blending the solution of PVC powders in THF and DBA, casting and evaporating in 
room temperature for several days. The sample became kind of soft gel-like dielectric elastomers with high transparency. The composition and size of each specimen are listed in Table 1.

Table 1 Composition and size of each specimen

\begin{tabular}{llll|lllc}
\hline $\begin{array}{l}\text { Sample } \\
\text { No. }\end{array}$ & $\begin{array}{l}\text { PVC: DBA } \\
(\mathrm{wt} \%)\end{array}$ & $\begin{array}{l}\text { Size } \\
\left(\mathrm{mm}^{2}\right)\end{array}$ & $\begin{array}{l}\text { Thickness } \\
(\mathrm{mm})\end{array}$ & $\begin{array}{l}\text { Sample } \\
\text { No. }\end{array}$ & $\begin{array}{l}\text { PVC: DBA } \\
(\mathrm{wt} \%)\end{array}$ & $\begin{array}{l}\text { Size } \\
\left(\mathrm{mm}^{2}\right)\end{array}$ & $\begin{array}{l}\text { Thickness } \\
(\mathrm{mm})\end{array}$ \\
\hline PVC30 & $10: 30$ & $5 \times 6$ & 0.57 & PVC70 & $10: 70$ & $5 \times 6$ & 0.38 \\
PVC50 & $10: 50$ & $5 \times 6$ & 0.51 & PVC90 & $10: 90$ & $5 \times 6$ & 0.55 \\
\hline
\end{tabular}

2. Compressive and Tensile measurements

The thermal mechanical analyzer was used in compressive and tensile measurements as shown in Fig.1. In order to investigate mechanical response under electrical field, a special setup with the electrodes on the sample table and base of the probe in sandwich was designed in a compressive test. The displacement and stress were obtained when an electric field was applied with $1000 \mathrm{~V} / \mathrm{mm}$, $1400 \mathrm{~V} / \mathrm{mm}, 1600 \mathrm{~V} / \mathrm{mm}, 1800 \mathrm{~V} / \mathrm{mm}$ and $2000 \mathrm{~V} / \mathrm{mm}$, respectively. After one cycle of applied electric fields from $1000 \mathrm{~V} / \mathrm{mm}$ to $2000 \mathrm{~V} / \mathrm{mm}$, the samples were recovered for 10 minutes and 20 hours, and then tested again without an electrical field. Thus, the mechanical behavior with/without the effect of electrical fields can be observed.

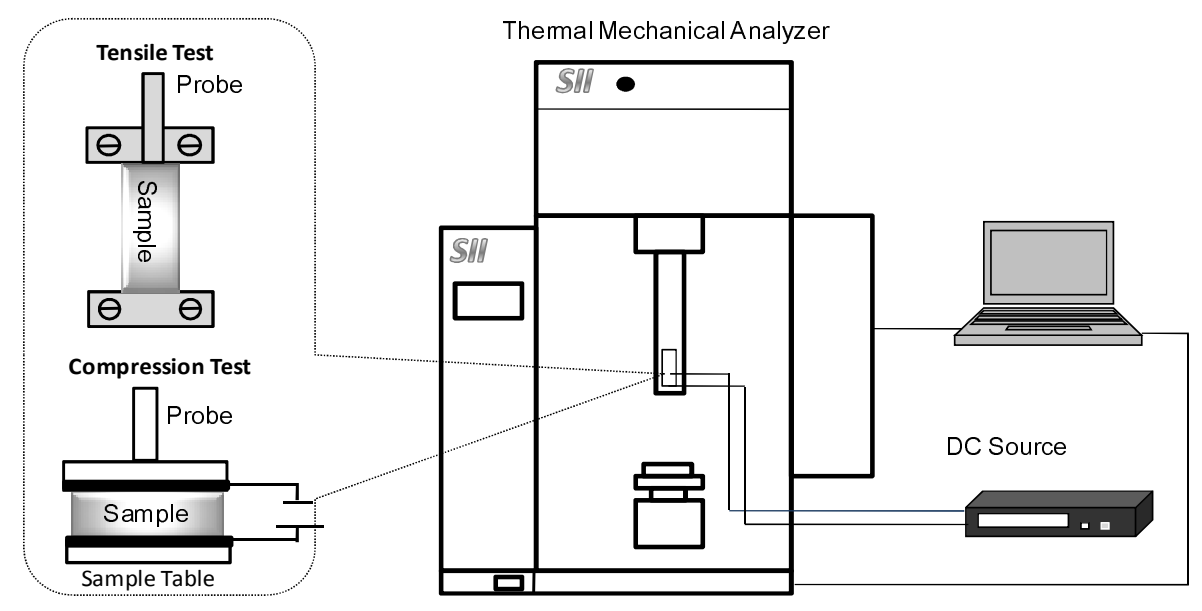

Fig.1. Schematic of thermal mechanical analyzer in compressive and tensile measurements.

3. Fluorescence microscope observation

Olympus (SZX12) Fluorescence Microscope was used for surface observation of PVC gels.

\section{Results and Discussions}

1. Influence of electrical stimulation on mechanical properties of gels

Fig. 2 shows the results of compressive test for each sample. For the original PVC samples, the strain curves are almost linear during the compressive loading process. However, after applied electric fields from $1000 \mathrm{~V} / \mathrm{mm}$ to $2000 \mathrm{~V} / \mathrm{mm}$, the compression curves consist of two parts with an obvious turning point at a low stress. It is shown that the recovery time also has an influence on the strain response behavior. It means that the experience of electrical fields has resulted in the change of PVC structure and mechanical property. It may be considered that a layer separation phenomenon was appeared in gels interior after applied electric fields. Their turn points also depend on plasticizer content of gels. The high DBA content of gel will result in a quick recovery. Thus, the recovery ability and the layer separation will depend on the order of plasticizer contents. The sample PVC90 with $90 \mathrm{wt} \%$ of DBA was almost recovered to the original strain behavior after 20 hours.

In the tensile test, we have found that initial Young's modulus became small after electrical stimulation but it had a tendency of recovery with the time. PVC30 shows much larger recovery ability than others after 20 hours (see Fig. 3), while the initial Young's modulus decreased with the 
increment of DBA contents. The results in Fig. 3 proved again that Young's modulus and recovery ability of PVC gels depend on the content of DBA.

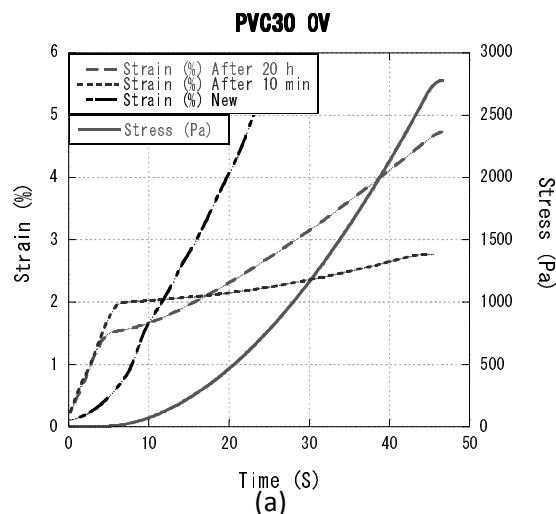

(a)

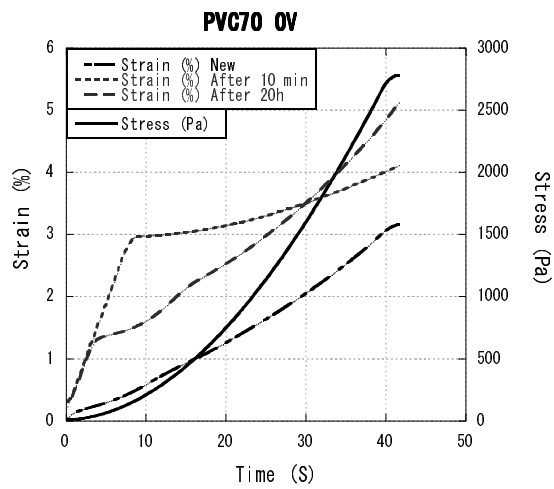

(c)

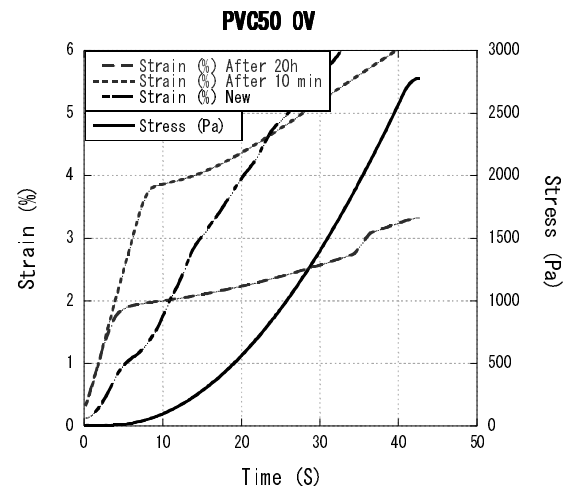

(b)

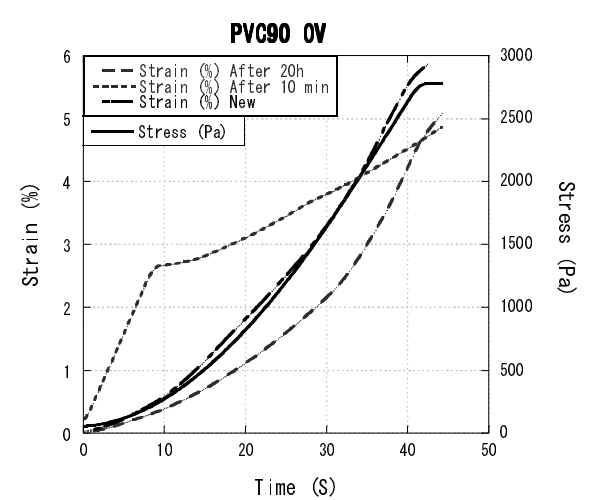

(d)

Fig. 2. The results of compressive test for new sample, after applied electric field for 10 minutes and 20 hours: (a) PVC30, (b) PVC50, (c) PVC70 and (d) PVC90.

2. Microstructure and a bending actuator

The fluorescence microscope observation was carried out to investigate the microstructure change before and after electrical field loading. In the Fig. 4(a), the DBA is distributed uniformly in the plasticized PVC. However, after applied the electrical fields from $1000 \mathrm{~V} / \mathrm{mm}$ to $2000 \mathrm{~V} / \mathrm{mm}$, we found that the cross-section becomes into two layers (see Fig. 4(b)). The two layers separation will be recovered partly when the sample is free of electrical field and its recovery ratio depends on the DBA contents in PVC gel. It is predicted that the mechanical property in these two layers should be quite different due to the great difference of mechanical property in PVC and DBA plasticizer. The tensile and compressive behaviors as mentioned in Fig. 2 and Fig. 3 depend greatly on this two layer separation. Layer 1 is near the anode and layer 2 is near the cathode when applying electrical fields. The thickness of layer 1 will be affected by sample thickness and the intensity of electrical field since Maxwell stress is a function of both them.

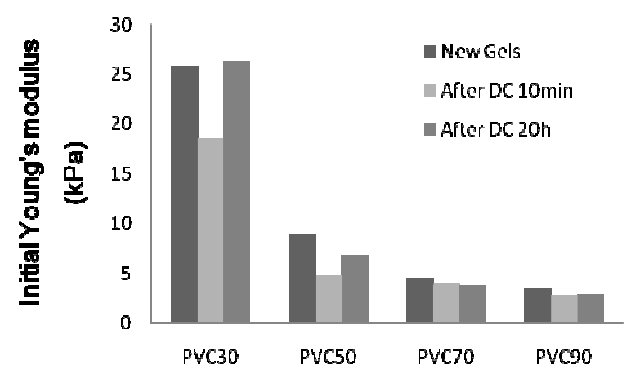

Fig.3. The results of tensile test.
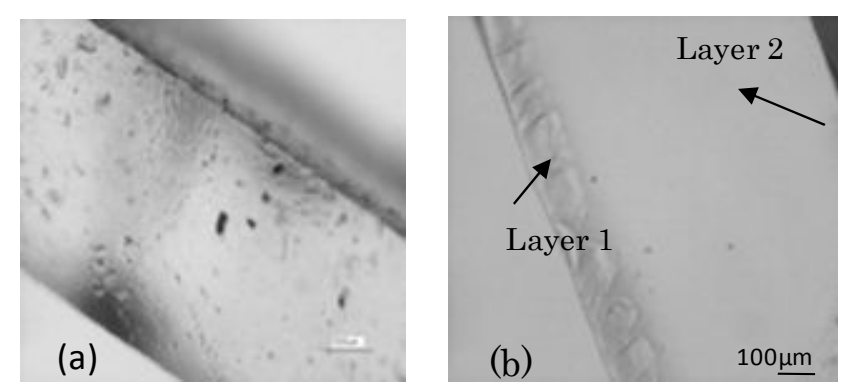

Fig. 4. The fluorescence microscope observation of PVC 90 gel section: before (a) and after applied electric fields. 
In the other hand, the formation of PVC gels is a gelation process that the plasticizer penetrates into PVC particles (primary) by swelling them, increasing their size, and gluing the particles together to form a homogeneous material. When temperature is lower than spinodal temperature, the homogeneous material may be separated to polymer-rich phase and polymer-poor phase as shown in Fig. 5(b) [4-6]. In the PVC gel, the polymer and plasticizer DBA both have strong polarization properties under an electrical stimulation. DBA moves to anode fast and PVC tends to have regular arrangement. The negative charges accumulate on gel surface near the anode. Thus, an attraction force will be generated between gel and anode [7] and it causes the polymer-poor phase moves to the anode and forms two-layer separation as observed in Fig. 4. Fig. 6 shows a bending actuator based on above two layer structures and Maxwell force. When an electrical field is applied on the PVC gel, layer separation occurs. The PVC gel will be compressed easily with the displacement, $\Delta \mathrm{d}$, since the layer1 may have a very low modulus. This compressive displacement accompanying with the attraction force occurred on the anode will make PVC gel bend to anode. The bending angle increased with the creep deformation progression (see Fig. 6).

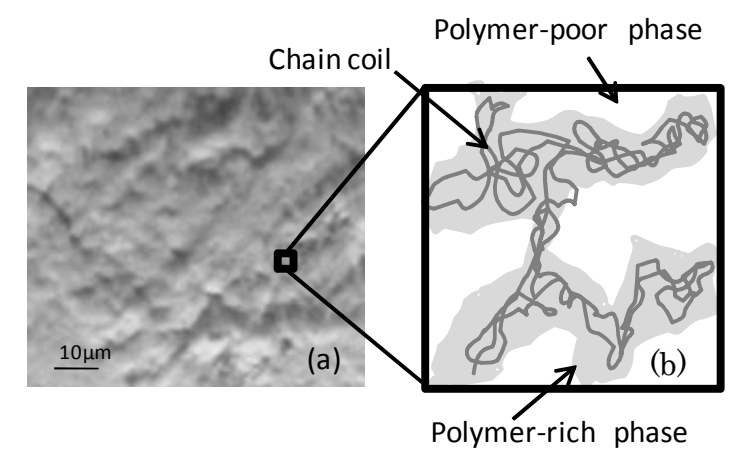

Fig. 5. Schematic of phase separation in PVC/DBA gels: (a)microstructure of gel. (b) a model of phase separation.

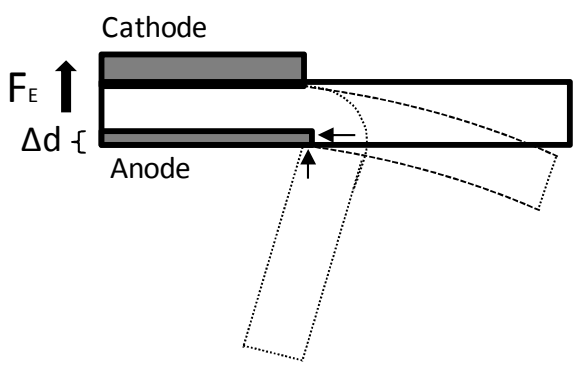

Fig. 6. Schematic of a bending actuator based on two layer structure and Maxwell stress.

\section{Conclusions}

This paper investigated the bending actuation and material memory effect of PVC gels. The layer separation of PVC gels under an electric field was found by fluorescence microscope observation. Layer 1 (near anode) is thinner and softer and its Young's modulus was much smaller than another layer. Thus, a creep deformation and bending actuation will be generated in anode. The anode was attracted into the gel with a compressive displacement, $\Delta \mathrm{d}$, and this causes the free part of the gel bend to the anode. Also their memory effect is maintained due to the layer separation. The actuation speed becomes faster and faster with electrical fields. However, their recoverability depends obviously on the content of plasticizer DBA. PVC 90 can be almost recovered after 20 hours.

For details of the relation between bending behaviors and gels memory effect, we will be reported in the next paper.

\section{Acknowledgment}

This work was partly supported by Grant-in-Aid for Global COE Program by the Ministry of Education, Culture, Sports, Science, and Technology, Japan.

\section{References}

[1] Ravi Shankar, Tushar K. Ghosh, Richard J. Spontak: Sensors and Actuators A, 151, 2009, 6-52.

[2] Md. Zulhash Uddin, Masashi Watanabe, Hirofusa Shirai and Toshihiro Hirai: Journal of Robotics and Mechatronics Vol. 14 No.2, 2002.

[3] Keiichi Yamamoto: master's thesis, Shinshu University, 2009.3, P90.

[4] Po-Da Hong, Che-Min Chou: Polymer 41, 2000, 8311-83320.

[5] Hassans Boudhani, Carole Laine, and Rene Fulchiron: Rheol Acta, 2007, 46, 825-838.

[6] Po-Da Hong and Che-Min: Macromolecules, 2000, 33 (26), 9673-9681.

[7] H. Xia, M. Takasaki and T. Hirai, submitted to Sensors and actuators A: physical. 